\section{Revised MERMAC test and questionnaire analysis system}

\author{
LAWRENCE M. ALEAMONI \\ University of Illinois at Urbana-Chamapign \\ Urbana, Illinois 61801
}

The MERMAC test and questionnaire analysis system (Aleamoni, 1971) was designed to assist instructors in developing valid and reliable tests and to provide rapid and meaningful feedback to the instructor and students. The MERMAC system was recently modified to operate on IBM Systems/360 and 370 with disk operating system (DOS).

Description. MERMAC is made up of two sets of programs: (a) utility (data manipulation) program, and (b) test and questionnaire analysis programs. The seven utility programs allow the user to copy, edit, match, merge, sequence, sort, and recode the input data. Generally, the purpose of these programs is to prepare the data for input to the test and questionnaire analysis programs. The six test and questionnaire analysis programs allow the user to: (1) Score item data and produce up to 40 subscores for each individual. Each item and response may be weighted to arrive at the scores. Any item may be included in more than one subscore and be weighted differently in each. (2) Take scores for a group of individuals and produce a frequency distribution and histogram, mean, median, standard deviation, Kuder-Richardson reliability, standard error of measurement, and Spearman-Brown prophecy for a reliability of .80 . In addition, individual raw scores, standard scores, and percentiles may be listed. Individual raw scores and standard scores can be weighted, summed, and the sum assigned a letter grade. All these data can be easily provided to the student.
(3) Return to each student a sheet containing his test score and a list of the items he missed with his responses and the correct responses. (4) Analyze his item data by providing a plot of the percentage of individuals responding to the keyed response by fifths of the total score distribution. For each item alternative, the proportion of individuals responding, a point biserial correlation, and the number responding to each alternative by fifths is provided. (5) Analyze his item data by using some external criterion rather than the keyeu' test score. (6) Summarize item data from questionnaires or tests with no known correct answers by 1. uviding a frequency distribution of responses, a veighted mean, and a standard deviation for each item. In addition, subscores may be generated with means, standard deviation, split-half reliabilities, and percentage of individuals responding to the contributing items. It is also possible to assign deciles to the item and subscore means based on a table look-up.

Computer and Language. The MERMAC system is written in basic assembly language (BAL) for both IBM System/360 Models 40 and above and the IBM System/370 Models 135 and above which have: (a) operating system (OS) with queued sequential access method (QSAM) support, and (b) disk operating system (DOS). It should be pointed out however, that the DOS version of MERMAC is not as flexible as the OS version.

Availability. Additional information about the program may be obtained free of charge from Lawrence M. Aleamoni, Measurement and Research Division, 307 Engineering Hall, University of Illinois at Urbana-Champaign, Urbana, Illinois 61801 .

\section{REFERENCE}

Aleamoni, L. M. MERMAC: A model and system for instructional test and questionnaire analysis, Behavior Research Methods \& Instrumentation, 1971, 3, 213-216. 